Journal of Patient-Centered

7-19-2021

\title{
Comprehensive Review and Update of Burning Eye Syndrome
}

\author{
Stephen Giacomazzi
}

Ivan Urits

Briggs Hoyt

Ashley Hubble

Elyse M. Cornett

Kyle Gress

Karina Charipova

Amnon A. Berger

Hisham Kassem

Alan D. Kaye

See next page for additional authors

Follow this and additional works at: https://aah.org/jpcrr

Part of the Alternative and Complementary Medicine Commons, Anesthesiology Commons, Eye Diseases Commons, Ophthalmology Commons, Sense Organs Commons, and the Therapeutics Commons

\section{Recommended Citation}

Giacomazzi S, Urits I, Hoyt B, Hubble A, Cornett EM, Gress K, Charipova K, Berger AA, Kassem H, Kaye AD, Viswanath O. Comprehensive review and update of burning eye syndrome. J Patient Cent Res Rev. 2021;8:255-60. doi: 10.17294/2330-0698.1813

Published quarterly by Midwest-based health system Advocate Aurora Health and indexed in PubMed Central, the Journal of Patient-Centered Research and Reviews (JPCRR) is an open access, peer-reviewed medical journal focused on disseminating scholarly works devoted to improving patient-centered care practices, health outcomes, and the patient experience. 


\section{Comprehensive Review and Update of Burning Eye Syndrome}

\section{Authors}

Stephen Giacomazzi, Ivan Urits, Briggs Hoyt, Ashley Hubble, Elyse M. Cornett, Kyle Gress, Karina Charipova, Amnon A. Berger, Hisham Kassem, Alan D. Kaye, and Omar Viswanath 


\title{
Comprehensive Review and Update of Burning Eye Syndrome
}

\author{
Stephen Giacomazzi, BS, ${ }^{1}$ Ivan Urits, MD, ${ }^{2}$ Briggs Hoyt, BS,${ }^{1}$ Ashley Hubble, BS, ${ }^{1}$ Elyse M. Cornett, \\ $\mathrm{PhD},{ }^{3}$ Kyle Gress, BS, ${ }^{4}$ Karina Charipova, BS, ${ }^{4}$ Amnon A. Berger, MD, PhD,${ }^{2}$ Hisham Kassem, MD, ${ }^{5}$ \\ Alan D. Kaye, MD, PhD, ${ }^{6}$ Omar Viswanath, $\mathrm{MD}^{7,8,9}$ \\ ${ }^{1}$ Creighton University School of Medicine, Omaha, NE; ${ }^{2}$ Department of Anesthesiology, Critical Care, and \\ Pain Medicine, Harvard Medical School, Beth Israel Deaconess Medical Center, Boston, MA; ${ }^{3}$ Department of \\ Anesthesiology, Louisiana State University Health Sciences Center, New Orleans, LA; ${ }^{4}$ Georgetown University \\ School of Medicine, Washington, DC; ${ }^{5}$ Department of Anesthesiology, Mount Sinai Medical Center, Miami Beach, FL; \\ ${ }^{6}$ Department of Anesthesiology, Louisiana State University Health Shreveport, Shreveport, LA; ${ }^{7}$ Valley Anesthesiology \\ and Pain Consultants - Envision Physician Services, Phoenix, AZ; ${ }^{8}$ Department of Anesthesiology, University of \\ Arizona College of Medicine-Phoenix, Phoenix, AZ; ${ }^{9}$ Department of Anesthesiology, Creighton University School of \\ Medicine, Omaha, NE
}

\begin{abstract}
Keratoconjunctivitis sicca ("dry eye") is a common (14\%-30\% of adults over age 48) though difficult to treat condition that causes both discomfort and disability with associated dryness, pain, and visual disturbances. Etiology is not clearly understood but is likely varied, with a subset of patients suffering from chronic neuropathic pain referred to as "burning eye syndrome." This review of existing literature summarizes the clinical presentation, natural history, pathophysiology, and treatment modalities of burning eye syndrome.

Chronicity of burning eye syndrome is likely secondary to increased nociception from the cornea, decrease in inhibitory signals, and nerve growth factor expression alterations. Treatment centers around symptomatic alleviation and reduction of inflammation. Conservative treatments focus on wellbeing and perception and include exercise, acupuncture, and cognitive behavioral therapy. Topical treatment consists of the anti-adhesion T-cell antagonist lifitegrast, corticosteroids, and cyclosporine; all have moderate efficacy and good safety. Autologous serum eye drops are a second-line topical that may promote corneal and neural healing on top of symptomatic relief. When these treatments fail, patients may trial neuromodulation with transcranial magnetic stimulation. Despite general treatment safety, more research is needed to develop novel approaches to this condition, possibly focusing more directly on the neurological component. (J Patient Cent Res Rev. 2021;8:255-260.)
\end{abstract}

Keywords burning eye syndrome; neuropathy; ocular pain; chronic pain; lifitegrast; neuromodulation

$\mathrm{D}$ ry eye disease (DED), or keratoconjunctivitis sicca, is a common eye condition with a wide variety of clinical presentations and is notoriously difficult to treat. ${ }^{1}$ DED can be defined as a multifactorial condition consisting of chronic sensations of dryness, pain, and visual disturbances in one or both eyes. ${ }^{2,3}$ There is currently no agreed-upon diagnostic criteria, which contributes significantly to its difficulty in treatment. ${ }^{1}$

Corresponding author: Ivan Urits, MD,

Beth Israel Deaconess Medical Center, 330 Brookline Ave., Boston, MA, 02215 (iurits@bidmc.harvard.edu)
The underlying mechanisms of DED have been a topic of discussion for many years. They have evolved to a current understanding consisting of multifactorial etiologies that can only partly be attributed to tear dysfunction.,3 Proposed underlying mechanisms include neurogenic inflammation, corneal sensory neuronal dysregulation, and tear film dysfunction, among others such as ocular surface inflammation. ${ }^{2-4}$ The traditional approach to treatment has been to address tear dysfunction, more specifically, to prevent the chronic cycle of damage from repeated dry eye exposure. ${ }^{5}$ However, it is well understood that this strategy has not adequately controlled symptoms in many patients. ${ }^{5}$ In light of this variability, attempts have been made to organize DED based on its possible underlying etiologies.

Recent literature has suggested that a subset of DED may be better conceptualized as a neuropathic pain disorder 
involving the eye..$^{2,3,5}$ This is largely in part to a subset of patients with features similar to neuropathic pain disorders such as spontaneous pain, hyperalgesia, and dysesthesia, and allodynia in relation to their eyes. ${ }^{2,5}$ "Burning eye syndrome" has been described as the subset of DED representing a systemic or localized neuropathic pain disorder involving the eye. ${ }^{2}$ Accordingly, recent treatment options for DED and its neuropathic subset, burning eye syndrome, have mirrored those used in chronic pain and neuropathic conditions, with some success. ${ }^{2,3}$

The diagnosis of burning eye syndrome remains one based on exclusion of other various causes of ocular pain. The objective of this article is to provide a comprehensive review of burning eye syndrome, with focus on clinical presentation, natural history, pathophysiology, and various treatment modalities.

\section{Signs and Symptoms}

Signs and symptoms of burning eye syndrome include those of dry eye such as pain, dysesthesias, and vision disturbances. The associated pain has been described as "burning," "aching," "tender," "foreign body-like," and "scratchy.", ",3,6 The neuropathic triad of "itch, pain, and dryness" also has been described in the setting of DED. ${ }^{7}$ These sensations can be spontaneously provoked or exacerbated by stress, cold, light, and dry air. ${ }^{3}$ Common visual disturbances experienced include blurry vision and poor visual acuity, which may be burdensome for patients. ${ }^{2}$ Overall, signs and symptoms of burning eye syndrome are variable and can be affected by surrounding anatomical abnormalities such as eyelid laxity, conjunctivochalasis, and Meibomian gland dysfunction. ${ }^{2}$

Patients can also present with comorbid neuropathic conditions, further reinforcing the possibility of a neuropathic mechanism underlying dry eye. Several studies have pointed to a relationship between chronic pain comorbidities and DED. ${ }^{4,5,8-10}$ A 2016 cross-sectional study of 425 patients by Vehof et al showed that chronic pain syndromes are associated with increased severity of DED symptoms..$^{10}$ In a 2017 study by Kalangara et al, a significant portion of 154 subjects endorsed descriptors that are commonly used in non-ocular neuropathic pain conditions. ${ }^{9}$ This study also found that DED signs did not correlate significantly with ocular pain severity, suggestive of a neuropathic pain mechanism. ${ }^{9}$

Epidemiological data analysis specifically applicable to burning eye syndrome is lacking. However, most ophthalmologists agree that DED is a tremendously prevalent condition with a wide range of clinical implications. Symptoms are estimated to affect $14 \%$ to $30 \%$ of the population 48 years of age and older.,
The economic burden of DED has been estimated at an indirect cost as high as $\$ 55.4$ billion annually, or $\$ 11,302$ per patient in the United States. ${ }^{2}$ The effect of DED can vary from a minor nuisance to debilitating. Results from Impact of Dry Eye on Everyday Life surveys have shown significant psychological and physical impairment, with severe scores even paralleling patients receiving dialysis or suffering from angina. ${ }^{2}$

\section{Risk Factors}

The risk factors for developing DED have been well described in cross-sectional studies and primarily include female gender and advanced age. ${ }^{11}$ Older studies have shown lifestyle factors such as smoking, caffeine use, and multivitamin use were found to be potentially significant risk factors for concurrent DED. In a retrospective study by Moss et al, 3722 patients were analyzed for risk factors concerning the prevalence of DED. ${ }^{12}$ History of arthritis, history of thyroid disease, history of gout, and diabetes were found to be the most important medical conditions associated with DED, with odds ratios of 1.91, 1.41, 1.42, and 1.38 , respectively.

Risk factors for burning eye syndrome have yet to be described in the literature. However, it is recognized that many neuropathic comorbidities exist in association with the neuropathic subset of DED, pointing at a possible connection. ${ }^{2,3,5}$ Comorbidities commonly seen in neuropathic pain disorders, namely depression and anxiety, also occur with DED (odds ratios of 2.92 and 2.80 , respectively). ${ }^{13}$

\section{Pathophysiology}

Like that of other conditions caused by DED, the pathophysiology of burning eye syndrome remains incompletely understood, in part because dry eye is a heterogenous condition whose variability likely stems from multiple genetic polymorphisms. ${ }^{2}$ Burning eye syndrome, a chronic neuropathic pain disorder that is considered by many to represent a subgroup of DED, is thought to result from a combination of peripheral and central sensitization. ${ }^{2}$ The process of sensitization is believed to involve highly sensitive, superficially located, unmyelinated corneal nociceptors that are programmed to respond to tear film evaporation and noxious mechanical forces. ${ }^{2,6}$ While tear dysfunction may be the initial insult in the development of DED and its subtypes, it does not define the entire pathophysiology; the persistent nature of the condition may be better explained by a more complex, acute-onchronic ocular sensory nerve injury that over time causes neuronal dysfunction and spontaneous ocular pain. ${ }^{2}$

Burning eye syndrome is considered by many to be a neuropathic pain disorder because it is a functional pain 
disorder characterized by somatosensory dysfunction and is disconnected in time from the initial tissue injury. ${ }^{3,5}$ The transition of injury from acute to chronic occurs through a self-propagating inflammatory cascade that culminates first in peripheral sensitization, which therefore decreases the activation thresholds of the corneal nociceptors through altered gene expression. ${ }^{2,3,14}$ This process of maladaptive peripheral neuronal plasticity is thought to lead to enhanced synaptic transmission and reduced postsynaptic activation thresholds that culminate in central sensitization. $^{3}$

It is believed that nerve growth factor (NGF) plays a critical role in the process of hypersensitization of the ocular surface. ${ }^{2}$ While the purpose of NGF at baseline is to defend the area of injury, its prolonged action is thought to lead to the hyperalgesia and allodynia seen in burning eye syndrome through altered neuronal healing and ectopic activity. NGF then, in turn, can promote expression of substance $\mathrm{P}$ and calcium gene-related peptide, which are implicated in nociceptive pain. It is hypothesized that while ascending pathways are hypersensitized, descending inhibitory pathways are dysregulated via the dysregulation of gamma aminobutyric acid (GABA) and glycine interneurons. The symptom of ocular surface dryness that is reported by some patients with the condition is believed to stem from the dysregulation of another type of neuron - the lacrimal functional unit. ${ }^{2}$

Unfortunately, a consensus is lacking in regard to structural abnormalities and morphologic changes that may underlie burning eye syndrome. ${ }^{3}$ Some patients who report DED symptoms have been shown to have reduced nerve densities; other studies have demonstrated a positive correlation between corneal sensitivity and nerve density. ${ }^{3}$ This discordance in the literature necessitates further research.

\section{Treatment}

Topical Anti-Inflammatories. Somatosensory dysfunction is thought to be one of the underlying factors involved in DED and caused by a release of inflammatory cytokines from both healthy and injured nerves and ongoing input of nociception. Topical anti-inflammatory therapies are often used in the treatment of neuropathic corneal pain to reduce the pathological signaling of nociceptors and promote physiologic healing of damaged nerves. ${ }^{3,15}$ Local therapies that have been shown to reduce local inflammation include topical corticosteroids, the T-cell antagonist lifitegrast, the immunomodulator cyclosporine, ${ }^{1,3}$ and autologous serum tears. Also, the use of ketorolac, a nonsteroidal anti-inflammatory drug, has been used to decrease pain and burning, especially in a perioperative setting. Punctal plugs, noninvasive silicone plugs placed in the lower punctum of the eyelid to close the tear duct, are another therapy shown to be effective in treating DED and may offer similar benefits in burning eye syndrome. ${ }^{16}$

Although some of these available options can alleviate the signs or the symptoms of burning eye syndrome, there is a necessity for treatments that address both the symptoms and the consequent ocular surface damage. ${ }^{17}$

Lifitegrast. Lifitegrast ophthalmic solution 5.0\%, known as a lymphocyte function-associated antigen 1 (LFA1) antagonist, is a new class of drug approved in the United States in 2016 for the treatment of burning eye syndrome. The mechanism of action involves blocking the interaction between the cell surface proteins, LFA-1, and intercellular adhesion molecule 1 (ICAM-1), which is believed to interrupt the inflammatory cascade associated with DED. ${ }^{17}$ Moreover, the main mechanism of this agent involves disrupting the cascade of inflammatory changes associated with $\mathrm{T}$ cells.

In a phase III randomized, double-masked, placebocontrolled trial that evaluated the efficacy and safety of lifitegrast versus placebo in patients with DED, lifitegrast was shown to improve symptoms of eye dryness significantly, as measured by eye dryness score, with improvement noted as early as day $14 .{ }^{18}$ Similarly, Holland et al systematically reviewed efficacy and safety data from prospective controlled trials of topical ophthalmic drugs for DED. ${ }^{17}$ In terms of both sign endpoint (inferior corneal staining score) and symptom endpoint (visual analog measure of eye dryness score) statistical superiority of those treated with lifitegrast versus a control group was demonstrated in 4 large $(\mathrm{N}>200)$ multicenter randomized controlled trials. Lifitegrast was generally well tolerated without any serious ocular treatment-emergent adverse events, with less than $5 \%$ of patients having to discontinue the medication due to relatively minor adverse events, the most common of which were mild to moderate instillation site irritation and site reaction. ${ }^{17}$

Cyclosporine. Cyclosporine A is a topical immunomodulatory agent approved by the U.S. Food and Drug Administration in 2003 for treating burning eye syndrome. It elicits anti-inflammatory properties by inhibiting cell-mediated reactions through the downregulation of interleukin-2 receptor expression and gene transcription. ${ }^{14,19}$ Wan at el systematically reviewed safety and efficacy of topical $0.05 \%$ cyclosporine in treating patients with DED among 12 randomized controlled trials incorporating 1367 patients. ${ }^{19}$ Compared to controls, patients using topical $0.05 \%$ cyclosporine eye drops twice daily had significantly improved objective 
and subjective outcomes. The endpoint included Ocular Surface Disease Index scores, longer tear film breakup time, reduced corneal fluorescein staining, and higher goblet cell densities. In addition to symptomatic improvement, it may slow down the disease progression of DED. ${ }^{19}$ In another meta-analysis, Zhou et al reported positive effects of topical cyclosporine on scores of breakup time and Schirmer test with anesthesia but no effect on any other factors. ${ }^{20}$ Multiple studies have reported minimal side effects associated with topical administration of cyclosporine. The most common adverse symptoms were of mild-moderate severity and included burning, stinging, and foreign body sensation, which resolved with cessation of treatment. ${ }^{14,19}$

Trophic Factors. Autologous serum eye drops have been used off label to treat signs of symptoms of burning eye syndrome, gaining popularity as a second-line treatment. ${ }^{3,15}$ In addition to serving as a lacrimal substitute, its other biochemical components mimic tears more closely, thus offering a potential advantage over traditional therapies..$^{15}$ Similar to tears, they are comprised of carbohydrates, lipids, and electrolytes. Specifically, they hold about 10 times more proteins, including albumin, fibronectin, and transferrin, mimicking innate eye secretions. ${ }^{14}$ They contain a variety of proneural factors like epidermal growth factors, NGF, and insulin-like growth factors, with concentrations ranging from $20 \%$ to $100 \%$, resulting in epithelial healing, increasing nerve density, and decreasing nerve tortuosity. ${ }^{4}$ Their effects are believed to be mediated in part by NGF, a neurotropin that regulates survival and differentiation of neurons by activation of tyrosine kinase A.,15

Autologous serum eye drops have been shown to be therapeutic in neurotrophic keratitis and corneal ulcers by possibly promoting the health and regeneration of corneal stroma, epithelium, and sensory nerves. ${ }^{3}$ Based on a Cochrane review of the current evidence, $20 \%$ autologous serum may provide some benefit in improving participant-reported symptoms in the short term (2 weeks), but the improvement was not observed through more extended periods of follow-up. Thus, the available data remains inconclusive regarding long term-efficacy. ${ }^{15}$ No effect was seen based on objective clinical measures of the ocular surface. ${ }^{15}$ Limitations for autologous serum eye drops include the requirement to harvest blood, limited availability, lack of insurance coverage, and need for storage in a freezer. ${ }^{3}$

Exercise/Acupuncture. Nonpharmacological methods - exercise, acupuncture, and peripheral and central stimulation - are essential adjuvants for the treatment of chronic neuropathic pain, which is the defining feature of burning eye syndrome. In patients with pain and contraindications to pharmacological interventions, these may even be used as primary treatments. ${ }^{3}$ Recent animal and human studies suggest that cardiovascular exercise inhibits pain pathways and can alter the perception of pain centrally. Additionally, exercise can increase brain neurotrophic factors, thereby contributing to neuroplasticity and neurorestoration. Given this evidence, it is suggested that patients participate in cardiovascular exercise for about 30-45 minutes at least 3-5 times per week. ${ }^{4}$

Acupuncture is one of the most popular forms of complementary and alternative medicine, and several studies have suggested its use for eye diseases. The rationale for its use is due to the claim that it modulates the autonomic nervous system and immune system, which subsequently regulates lacrimal gland function. ${ }^{4}$ There is also increasing evidence that acupuncture stimulates endogenous opioid mechanisms and stimulates gene expression and neuropeptides. ${ }^{4}$

Goyal et al assessed the effectiveness of acupuncture as a treatment option for conditions of dry eye, which included 6 randomized controlled trials comparing acupuncture to conventional treatment. Results supported the efficacy of acupuncture on DED, as well as acupuncture plus artificial tears, as compared to artificial tears alone. However, the number of trials, total sample size, and methodological quality was too low to make firm conclusions. ${ }^{4} \mathrm{Kim}$ et al updated the meta-analysis and conducted subgroup analysis, concluding that acupuncture was more effective than artificial tears in treating DED but showed high heterogeneity, as in prior studies. ${ }^{21}$ The subgroup analysis demonstrated that long-term ( $>1$ month) and less frequent $(<3$ times a week) application might be more effective than short-term or intensive treatments. ${ }^{21}$

Cognitive Behavioral Therapy. Cognitive behavioral therapy decreases maladaptive thoughts and behaviors, reduces anxiety associated with chronic pain, and improves coping mechanisms. This makes it a potentially important component in treating patients with chronic burning eye syndrome. ${ }^{3}$ In one retrospective study, mental disorders in refractory dry eyes were analyzed after the evaluation of questionnaires in 110 patients. Anxiety or depression had been diagnosed in $52.7 \%$ of patients (anxiety in $21.8 \%$, depression in $15.3 \%$, both in $14.5 \%$ ) and vegetative dystonia in $25.4 \%$. Only $22.7 \%$ reported no diagnosis of a mental illness, demonstrating that therapy-resistant dry eyes are often associated with mental health disorders. ${ }^{22}$ The underlying mechanisms may be related to the production of proinflammatory cytokines in chronic depression. Furthermore, depressed mood can lower the threshold for perception of discomfort or pain caused by burning eye syndrome. 
Somatization is a frequently reported condition in depression and may predispose the development of DED. ${ }^{23}$ Rosenthal et al found that many patients with long-standing ocular facial pain conveyed substantial deterioration of their cognitive and executive functions and working memory, consistent with reports in fibromyalgia patients. Moreover, it has been shown that patients diagnosed with DED are burdened by low self-assessment scores on how their health and well-being affects their ability to complete activities of daily living. With this perspective, the management of patients with burning eye syndrome should consider the bidirectional effects of depression and chronic pain. ${ }^{6}$ This supports the notion that psychological therapies, such as cognitive-behavioral therapy, can potentially benefit patients with DED. However, there are currently no reports on the psychological treatment of dry eye, corneal pain, or ocular surface pain..$^{24}$

Direct Nerve Treatments. Patients who have been refractory to the aforementioned treatments could undergo a trial of transcranial magnetic stimulation. This noninvasive method causes neurons in the brain to depolarize or hyperpolarize. High-frequency repetitive transcranial magnetic stimulation of the brain regions that correspond to the body parts in pain may be an effective analgesic. A meta-analysis evaluating the analgesic effect found it to be more effective in suppressing central rather than peripheral neuropathic corneal pain. ${ }^{4}$

Sivanesan et al used ocular transcutaneous electrical nerve stimulation to alleviate symptoms of ocular discomfort, photophobia, and dryness. This direct approach significantly reduced the mean pain intensity and the light sensitivity in both eyes following treatment, indicating that transcutaneous electrical nerve stimulation may provide analgesia in patients with burning eye symptoms as it does for other chronic pain conditions. ${ }^{25}$

Scrambler therapy is another option for controlling chronic neuropathic pain and works by interfering with pain signal transmission. By mixing nonpain information with the pain signals into the nerve fibers, the nervous system becomes confused and unable to sense pain. Multiple studies have reported that this therapy may be more successful in relieving neuropathic pain than previously outlined medications. ${ }^{4}$ Goyal et al emphasized that applying multiple treatment strategies often yielded better clinical success. ${ }^{4}$

Neuromodulation involves the stimulation or administration of medications directly into the nervous system, allowing focused delivery of modifying agents to improve neural function in targeted areas. Implantable and nonimplantable devices deliver electrical, chemical, or other agents to reversibly modify brain and nerve cell activity. ${ }^{4}$ In one case study, two new neuromodulatory approaches were applied to a patient with recalcitrant corneal neuropathic pain secondary to laser in situ keratomileusis surgery. ${ }^{26}$ An electrode was implanted for electrical stimulation of the trigeminal ganglion, resulting in complete resolution of symptoms until lead migration caused a recurrence of pain. Subsequently, implantation of an intrathecal infusion for fentanyl and bupivacaine delivery at the cervical (C1-C2) level controlled the patient's symptoms and provided excellent pain relief at 1 year. ${ }^{26}$ In recent literature, Dieckmann et al discussed that neuroregenerative approaches to treat ocular pain syndromes, such as with direct autologous serum tears, plasma rich in growth factors, platelet-rich plasma, autologous plasma, and NGF, have shown promising results. ${ }^{27}$

\section{Summary}

Dry eye disease is a common, disabling condition that can present with symptoms of dryness, pain, and visual disturbances in either one or both eyes. The etiology is still debated but is likely secondary to tear dysfunction. The presence of pain, hyperalgesia, and dysesthesia bring forward the possibility that a subset of this dry eye population can be grouped by their chronic neuropathic pain symptoms; these patients are characterized as having burning eye syndrome. This is strengthened by studies showing that patients describe the pain using descriptors common to neuropathic pain over other ocular conditions. Research findings most often assign blame for the transition from acute to chronic injury on an inflammatory cascade that results in nociceptive activation of corneal sensory nerves, through genetic alterations. Nerve growth factor is likely involved, and the altered expression results in increased nociceptive activation and decreased GABA inhibition.

Treatment of burning eye syndrome is focused on both symptomatic alleviation and reduction of inflammation. Topical options include corticosteroids, lifitegrast, and cyclosporine. Lifitegrast was recently shown to be both safe and effective, while cyclosporine provides some relief with relatively few side effects. Autologous serum eye drops are a second-line topical treatment that adds epidermal healing. Nonpharmacological therapies targeting the perception of pain and discomfort include exercise, acupuncture, and cognitive behavioral therapy.

Patients who fail conservative and topical treatment may also attempt neuromodulation with transcranial magnetic stimulation, transcutaneous electrical nerve stimulation, or direct delivery of therapy to neuronal targets. Further evidence is needed to reveal the overall efficacy and safety of these novel therapies. 


\section{Patient-Friendly Recap}

- Patients with dry eye disease who experience chronic pain generated by their nervous systems are characterized as having burning eye syndrome.

- Authors reviewed clinical signs, symptoms, risk factors, disease physiology, and treatments for burning eye syndrome.

- Topical options for symptom relief include antiinflammatories, immunosuppressant drugs, and serum eye drops. Exercise, acupuncture, cognitive behavioral therapy, and newer neuromodulation techniques also have been shown to have therapeutic benefits.

\section{Author Contributions}

Study design: all authors. Data acquisition or analysis: all authors. Manuscript drafting: all authors. Critical revision: all authors.

\section{Conflicts of Interest}

None.

\section{References}

1. Jacobs DS. Diagnosis and treatment of ocular pain: the ophthalmologist's perspective. Curr Ophthalmol Rep. 2017;5:271-5. CrossRef

2. Kalangara JP, Galor A, Levitt RC, Felix ER, Alegret R, Sarantopoulos CD. Burning eye syndrome: Do neuropathic pain mechanisms underlie chronic dry eye? Pain Med. 2016;17:746-55. $\underline{\text { CrossRef }}$

3. Galor A, Moein HR, Lee C, et al. Neuropathic pain and dry eye. Ocul Surf. 2018;16:31-44. CrossRef

4. Goyal S, Hamrah P. Understanding neuropathic corneal pain gaps and current therapeutic approaches. Semin Ophthalmol. 2016;31:59-70. $\underline{\text { CrossRef }}$

5. Levitt AE, Galor A, Chowdhury AR, et al. Evidence that dry eye represents a chronic overlapping pain condition. Mol Pain. 2017;13:1744806917729306. CrossRef

6. Rosenthal P, Borsook D. Ocular neuropathic pain. $\mathrm{Br} J$ Ophthalmol. 2015;100:128-34. CrossRef

7. Andersen HH, Yosipovitch G, Galor A. Neuropathic symptoms of the ocular surface: dryness, pain, and itch. Curr Opin Allergy Clin Immunol. 2017;17:373-81. CrossRef

8. Schuster AK, Wettstein M, Gerhardt A, Eich W, Bieber C, Tesarz J. Eye pain and dry eye in patients with fibromyalgia. Pain Med. 2018;19:2528-35. CrossRef

9. Kalangara JP, Galor A, Levitt RC, et al. Characteristics of ocular pain complaints in patients with idiopathic dry eye symptoms. Eye Contact Lens. 2017;43:192-8. CrossRef

10. Vehof J, Sillevis Smitt-Kamminga N, Kozareva D, Nibourg SA, Hammond CJ. Clinical characteristics of dry eye patients with chronic pain syndromes. Am J Ophthalmol. 2016;166:203-4. $\underline{\text { CrossRef }}$
11. Nebbioso M, Del Regno P, Gharbiya M, Sacchetti M, Plateroti $\mathrm{R}$, Lambiase A. Analysis of the pathogenic factors and management of dry eye in ocular surface disorders. Int $\mathrm{J} \mathrm{Mol}$ Sci. 2017;18:1-19. CrossRef

12. Moss SE, Klein R, Klein BE. Prevalance of and risk factors for dry eye syndrome. Arch Ophthalmol. 2000;118:1264-8. CrossRef

13. Wan KH, Chen LJ, Young AL. Depression and anxiety in dry eye disease: a systematic review and meta-analysis. Eye (Lond). 2016;30:1558-67. CrossRef

14. Drew VJ, Tseng CL, Seghatchian J, Burnouf T. Reflections on dry eye syndrome treatment: therapeutic role of blood products. Front Med (Lausenne). 2018;5:33. CrossRef

15. Pan Q, Angelina A, Marrone M, Stark WJ, Akpek EK. Autologous serum eye drops for dry eye. Cochrane Database Syst Rev. 2017;2(2):CD009327.

16. Song JS, Woo IH, Eom Y, Kim HM. Five misconceptions related to punctal plugs in dry eye management. Cornea. 2018;37 Suppl 1:S58-61. CrossRef

17. Holland EJ, Darvish M, Nichols KK, Jones L, Karpecki PM. Efficacy of topical ophthalmic drugs in the treatment of dry eye disease: a systematic literature review. Ocul Surf. 2019;17:412-23. CrossRef

18. Holland EJ, Luchs J, Karpecki PM, et al. Lifitegrast for the treatment of dry eye disease: results of a phase III, randomized, double-masked, placebo-controlled trial (OPUS3). Ophthalmology. 2017;124:53-60. CrossRef

19. Wan KH, Chen LJ, Young AL. Efficacy and safety of topical $0.05 \%$ cyclosporine eye drops in the treatment of dry eye syndrome: a systematic review and meta-analysis. Ocul Surf. 2015;13:213-25. CrossRef

20. Zhou XQ, Wei RL. Topical cyclosporine A in the treatment of dry eye: a systematic review and meta-analysis. Cornea. 2014;33:760-7. CrossRef

21. Kim BH, Kim MH, Kang SH, Nam HJ. Optimizing acupuncture treatment for dry eye syndrome: a systematic review. $B M C$ Complement Altern Med. 2018;18:145. $\underline{\text { CrossRef }}$

22. Nepp J. [Psychosomatic aspects of dry eye syndrome]. Ophthalmologe. 2016;113:111-9. CrossRef

23. Han SB, Yang HK, Hyon JY, Wee WR. Association of dry eye disease with psychiatric or neurological disorders in elderly patients. Clin Interv Aging. 2017;12:785-92. CrossRef

24. Steenholdt C, Brynskov J, Thomsen OØ, et al. Individualised therapy is more cost-effective than dose intensification in patients with Crohn's disease who lose response to anti-TNF treatment: a randomised, controlled trial. Gut. 2014;63:919-27. CrossRef

25. Sivanesan E, Levitt RC, Sarantopoulos CD, Patin D, Galor A. Noninvasive electrical stimulation for the treatment of chronic ocular pain and photophobia. Neuromodulation. 2018;21:727-34. CrossRef

26. Sayegh RR, Sweet JA, Miller JP, Hayek SM. Electrical stimulation of the trigeminal ganglion and intrathecal drug delivery systems for the management of corneal neuropathic pain. Cornea. 2016;35:576-7. CrossRef

27. Dieckmann G, Goyal S, Hamrah P. Neuropathic corneal pain: approaches for management. Ophthalmology. 2017;124(11S):S34-47. CrossRef

(C) 2021 Advocate Aurora Health, Inc. 